\title{
Saccades elicit obligatory allocation of visual working memory
}

\author{
Na Shao, Jie Li, Rende Shui, Xiaojie Zheng, Jiangang Lu, and Mowei Shen \\ Zhejiang University, Hangzhou, China
}

\begin{abstract}
In daily life, visual working memory (VWM) typically works in contexts in which people make frequent saccades. Here, we investigated whether people can effectively control the allocation of VWM when making a saccade. Subjects were required to make an intervening saccade in the process of a memory task. The saccade target was either a to-be-remembered item or an extra, not-to-be-remembered item. The results showed that memory performance was poorer when a saccade was made to the extra, not-to-be-remembered item, regardless of its similarity to the memory item(s). In contrast, when memorizing the items while remaining fixated, subjects had similar memory performance whether an extra, not-to-be-remembered item was present or not. Taken together, these results demonstrated that volitional control over VWM allocation is greatly impaired when a saccade is made, indicating that VWM contains an automatic part that cooperates with eye movements and is allocated to a saccade target obligatorily.
\end{abstract}

Our ability to temporarily maintain in memory what we have seen is highly limited (Cowan, 2001; Luck \& Vogel, 1997). This limitation necessitates effective control or selective allocation of visual working memory (VWM; Cowan $\&$ Morey, 2006). Previous research has demonstrated that we are indeed able to control which information is held in VWM on the basis of task demands (Vogel, McCollough, \& Machizawa, 2005). The control can be exerted in both the early and late stages of VWM, through the attenuation of perceptual processing of distractors (Sreenivasan \& Jha, 2007), the attentional enhancement of the stimuli that most possibly will be tested (Griffin \& Nobre, 2003), or the filtration of irrelevant information directly from VWM (Nasr, Moeeny, \& Esteky, 2008). The ability to control VWM correlates with other cognitive and developmental processes: Individuals with higher VWM capacity have better control of VWM (Vogel et al., 2005); adults have a stronger ability to ignore distraction than children have (Olesen, Macoveanu, Tegner, \& Klingberg, 2007).

In the present study, we investigated the control of VWM under circumstances involving saccades. This is an important issue because VWM processing in daily life typically involves active visual sampling, since people make saccades approximately three times per second in order to fixate on important or interesting objects (Ross, Morrone, Goldberg, \& Burr, 2001). In previous studies, the control of VWM has been demonstrated mostly when people were remaining fixated. Considering the impact of eye movements on cognitive processes (Ballard, Hayhoe, \& Pelz, 1995; Melcher \& Colby, 2008; Prime, Tsotsos, Keith, \& Crawford, 2007), it is unclear whether people are still able to effectively control the allocation of VWM when making a saccade. One recent study pertinent to this issue showed that VWM allocation could be altered by a saccade (Bays \& Husain, 2008). In this study, subjects' memory for the location or orientation of multiple visual objects after a brief disappearance of the stimulus array was tested, with or without an intervening saccade. The results showed that, overall, memory performance was similar in the saccade and fixation conditions, indicating that a saccade did not reduce the total VWM available. More important, a further analysis of memory performance in the saccade condition revealed that the upcoming saccade target was remembered better than other objects. This result indicated that a saccade did affect the allocation of VWM, biasing it toward the saccade target. However, in Bays and Husain's (2008) study, subjects were required to always remember all the items, including the saccade target. They did not need to voluntarily control the allocation of VWM according to the task. Therefore, it is unknown whether the saccade-elicited allocation could have been controlled if required.

The aim of the present study was to examine whether one still has the ability to control the allocation of VWM when making a saccade. Specifically, does a saccade target automatically obtain allocation of VWM regardless of task demand (obligatory allocation), or can one effectively allocate VWM to task-related items (controlled allocation)?

To investigate the controllability of VWM in the saccade condition, we manipulated the saccade target to be either relevant or irrelevant to the memory task. ${ }^{1}$ Under a modified version of the change detection task (Bays \& Husain, 2008), subjects' ability to remember the orientations 
of some arrows was estimated after a brief disappearance of the stimulus array, with an intervening saccade to one of the to-be-remembered arrows (saccade-to-memoryrelated-arrow [SMRA] condition) or to a not-to-beremembered arrow (saccade-to-memory-unrelated-arrow [SMUA] condition). Memory precision for the items was estimated in order to reveal the allocation of VWM.

The question of interest was that, when making a saccade to an extra, not-to-be-remembered item, will people still obligatorily allocate VWM to the saccade target, even at the expense of memory performance for the to-be-remembered items? If they will, the total VWM allocated to the tobe-remembered items should be reduced; thus, subjects' memory precision for to-be-remembered items should be worse when they make a saccade to an extra, not-tobe-remembered object (SMUA condition) than when they make a saccade to one of the memory items (SMRA condition). On the other hand, if VWM can be controlled so as to be allocated merely to the to-be-remembered items, but not to the extra, memory-unrelated saccade target, memory precision should be similar in the two conditions.

\section{EXPERIMENT 1}

In Experiment 1, we tested whether subjects could volitionally avoid allocating VWM to a memory-unrelated saccade target. The subjects' ability to remember the orientation(s) of arrow(s) ${ }^{2}$ was tested after a brief disappearance of the stimulus array, with an intervening saccade toward one of the memory items (see Figure 1; SMRA condition) or toward the extra, not-to-be-remembered item (SMUA condition), in separate blocks.

\section{Method}

Subjects. Twelve subjects (22-26 years of age; 5 of them female and 7 male) with normal vision participated in this experiment. One

A

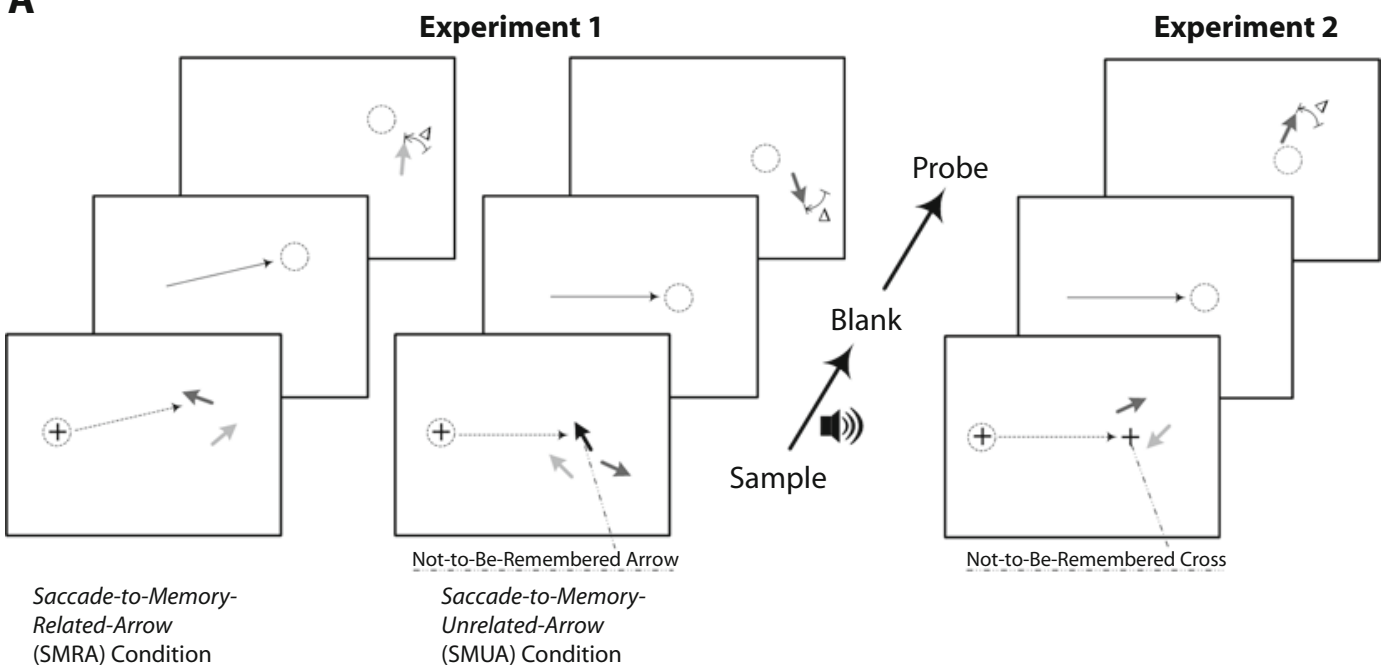

B

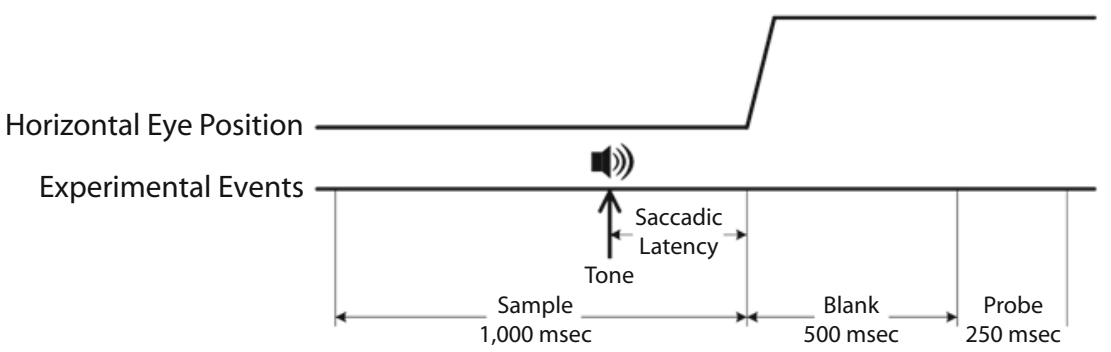

Figure 1. (A) Schematic illustration (not drawn to scale) of the stimuli and experimental designs used in Experiments 1 and 2. Colored (represented by gray) arrows were memory items, whereas the black arrow in the saccade-to-memory-unrelated-arrow (SMUA) condition in Experiment 1 and the black cross in Experiment 2 were irrelevant for the memory task. Before the offset of the sample display, a tone was sounded. At the tone, the subject made a saccade toward the saccade target. In the saccade-to-memory-related-arrow (SMRA) condition (Experiment 1), the saccade target was an arrow of a prespecified color (here blue, represented by dark gray); in the SMUA condition (Experiment 1), the saccade target was a not-to-be-remembered black arrow; and in Experiment 2, the saccade target was a not-to-be-remembered black cross. A 500-msec blank appeared at around the starting time of the saccade. Then a randomly chosen arrow was redisplayed and rotated through an angle $\Delta$, and the subject reported the direction of rotation, either clockwise or counterclockwise. Dashed circles and arrows indicate gaze position and saccadic direction, respectively. The number of the memory items is two in all the examples above. (B) Temporal structure of a trial. The upper trace illustrates the subject's schematic horizontal eye movement trajectory; the lower line shows critical experimental events according to the time line. 
of the subjects was an author, and the others were healthy volunteers who were naive as to the purpose of the experiment.

Stimuli and Apparatus. The stimuli consisted of randomly oriented colored arrows presented against a gray background. The length of an arrow was $1.25^{\circ}$ of visual angle. Stimulus colors were randomly selected without repetition on each trial from a set of highly discriminable colors (white, black, red, green, blue, yellow, and cyan). The number of to-be-remembered arrows in the sample display was one, two, four, or six and varied from trial to trial. The to-be-remembered arrows were randomly arranged within an invisible square $\left(9^{\circ} \times 9^{\circ}\right)$ centered $10^{\circ}$ horizontally from a fixation cross that deviated $7^{\circ}$ horizontally from the screen center and randomly alternated left or right on each trial. The minimum separation between arrows was $3^{\circ}$. The saccade target was one of the to-be-remembered arrows with a prespecified color in the SMRA condition, whereas it was the not-tobe-remembered black arrow always displayed at the center of the invisible square in the SMUA condition (see Figure 1).

The stimuli were displayed at a resolution of $1,024 \times 769$ pixels on a 22-in. Mitsubishi Diamond Pro 2070SB color monitor driven by a display controller (Cambridge Research System VSG ViSaGe; Cambridge Research Systems, Cambridge, U.K.) with a $140-\mathrm{Hz}$ refresh rate (frame duration $=7.14 \mathrm{msec}$ ), under the control of a Pentium IV personal computer. At a viewing distance of $57 \mathrm{~cm}$, the screen subtended $40^{\circ} \times 30^{\circ}$. A lookup table in the software was used to linearize the intensity response of the screen phosphors at an 8-bit luminance resolution.

Movements of one eye of the subjects were measured with an infrared high-speed video eyetracker (HS-VET, Cambridge Research Systems) at a sampling rate of $200 \mathrm{~Hz}$. Acquisition of ocular data was triggered by a frame synchronization signal from the display controller to allow accurate alignment of eye movements and presentation of the stimuli. Head movements were minimized with chin- and headrests. The system was calibrated at the beginning of the test session and after every break. The experimenter was able to monitor the eye position online and interrupt the session for recalibration when necessary. Saccades were detected by using $2^{\circ}$ movement and $50 \%$ sec velocity criteria. Each observer's average saccade latency was measured at the beginning of the experiments.

Procedure and Design. All the trials began with the presentation of a fixation cross. Once the subject was fixating the cross, the sample display was presented for $1,000 \mathrm{msec}$, followed by a blanking period of $500 \mathrm{msec}$ and presentation of a probe display for $250 \mathrm{msec}$. The probe display consisted of the reappearance of a randomly chosen to-be-remembered arrow from the sample display, but rotated clockwise or counterclockwise $5^{\circ}, 20^{\circ}$, or $45^{\circ}$. The subjects were asked to report the direction of the rotation with a buttonpress response.

The memory task was accompanied by an intervening saccade. During the sample display, an auditory go signal sounded, at which the subject made a saccade from the fixation cross toward the saccade target. The interval between the auditory go signal and the offset of the sample display matched the estimate of average saccade latency obtained from previous saccade trials. In this manner, the subject's eyes typically started moving at around the offset of the sample display.

The major independent variable in this experiment was type of saccade target. In the SMRA condition, the saccade target was one of the to-be-remembered colored arrows (prior to the experiment, each subject was randomly allocated a color that would indicate the saccade target on each trial), whereas in the SMUA condition, the saccade target was an extra, not-to-be-remembered black arrow at the center of the arrow display square. The subjects were informed clearly that the memory-unrelated saccade target in the SMUA condition would not be probed through all the trials and that the chance that each to-be-remembered item (including the saccade target in the SMRA condition, but not in the SMUA condition) in the sample display would be probed was the same. The two conditions were tested in different blocks, and the order of the blocks was counterbalanced across subjects. Four types of number of tobe-remembered items (one, two, four, and six) in the sample display were randomly mixed together, with an equal proportion in each condition.

Whether a trial was included in the analysis was determined by the subject's saccade parameters on that trial. An included trial had to meet the four following criteria: (1) The subject initiated the saccade after the onset of the auditory go signal; (2) the onset of the saccade was, at most, $35 \mathrm{msec}$ (minimum duration of a saccade in the present experiments) before the offset of the sample display, to make sure that sample items disappeared when the eyes landed; (3) the onset of the saccade was, at most, $600 \mathrm{msec}$ after the onset of the auditory go signal, so that the saccade latency would not be too long and the probe display would not appear before the saccade was finished; (4) the subject had not blinked during the saccade. Each condition continued to run until at least 192 included trials were completed per condition for each subject. The percentages of included trials were acceptable and consistent among different conditions, as revealed by additional analyses of the experimental data, which demonstrated the effectiveness of the experimental method (see Appendix A for more information).

\section{Results and Discussion}

The subjects' responses varied probabilistically with the magnitude of the change to the probe arrow (see Figure 2). The error in the stored representation of the original stimulus followed the Gaussian distribution (McNicol, 1972; Wickens, 2002), as also was shown by Bays and Husain (2008). A probit regression $\operatorname{model}^{3}$ was used to
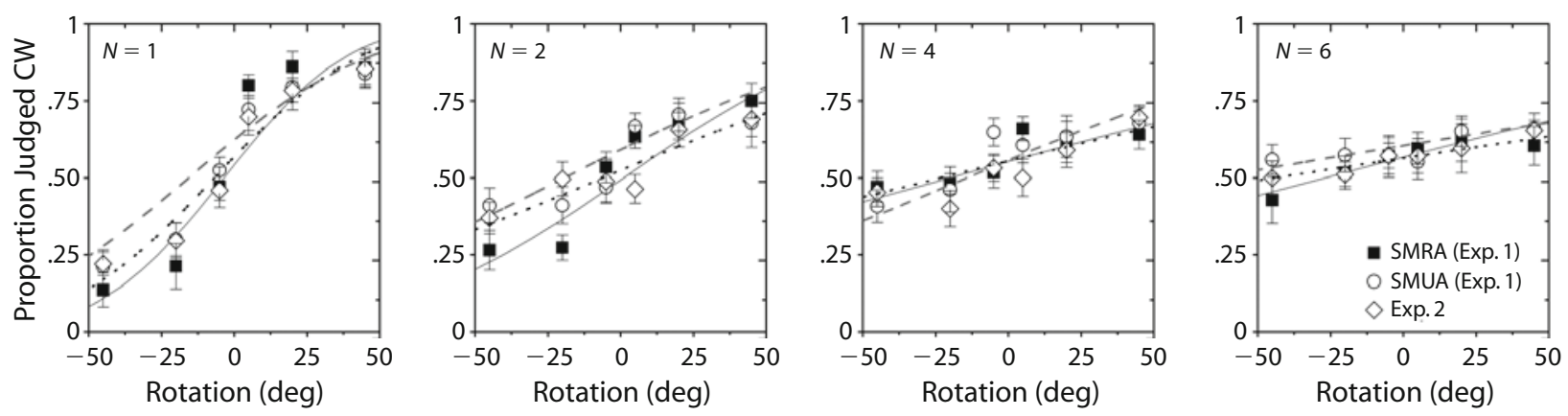

Figure 2. Proportion of orientations (rotations) judged clockwise $(\mathrm{CW})$ as a function of the actual rotation and number of memory items $(N)$. Curves indicate cumulative Gaussian distributions fitted to the response data. Data for the saccade-to-memory-related-arrow (SMRA) and saccade-to-memory-unrelated-arrow (SMUA) conditions in Experiment 1 are fitted by the solid line and dotted line, respectively, and those for Experiment 2 are fitted by the dashed line. Error bars indicate $\pm 1 S E$. 
estimate the parameters (mean and standard deviation) of the cumulative Gaussian distribution that best fit the relationship between response probability and stimulus rotation in each condition (Bays \& Husain, 2008).

The cumulative Gaussian distribution provides two parameters - namely, the mean and the standard deviation. In the present study, any discrimination bias was indicated by the mean of the fitted Gaussian $(\mu)$, whereas memory precision was determined by the reciprocal of the standard deviation $(1 / \sigma)$. Smaller standard deviations correspond to steeper slopes of the cumulative Gaussian distribution, showing that response stabilities are higher. That is, the subject is able to judge the changes of the orientations more stably, indicating that memory precision is greater. In the data analyses, we focused on the influence of the conditions on precision (reciprocal of the standard deviation of probit models) in order to reveal VWM allocation under different circumstances. The goodnesses of fit in the two conditions and following the experiments/conditions were acceptably good (see Appendix B for details).

We performed a $2 \times 4$ repeated measures ANOVA on memory precision, with type of saccade target (memoryrelated arrow vs. memory-unrelated arrow) and number of memory items (one, two, four, or six) as within-subjects factors. In this and all the following data analyses, the Greenhouse-Geisser correction was applied when appropriate to correct for possible violation of the ANOVA assumption of sphericity. The text gives corrected $p$ values.

Figure 3 shows the precision of VWM in the SMRA and SMUA conditions. The main effect of type of saccade target was significant $\left[F(1,11)=9.032, p=.012, \eta_{\mathrm{p}}^{2}=\right.$ $.510]$, due to the lower precision in the SMUA condition than in the SMRA condition. There was also a significant main effect of number of memory items $[F(3,33)=$ $\left.16.893, p=.001, \eta_{\mathrm{p}}^{2}=.606\right]$, reflecting a decrease in the precision of VWM as a function of number of to-

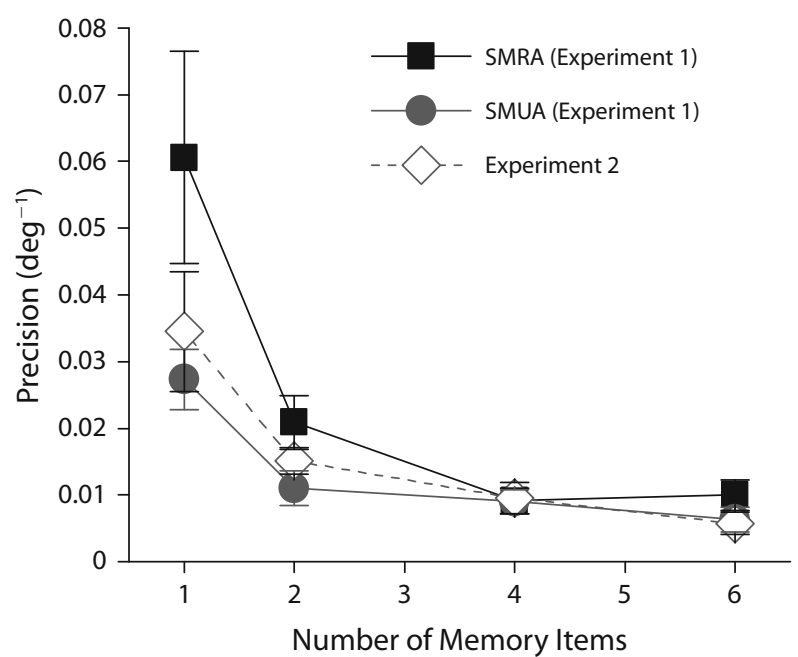

Figure 3. Memory precision (determined by the reciprocal of the $S D$ of the fitted Gaussian) as a function of number of memory items and of experimental conditions in Experiments 1 and 2. Error bars indicate $\pm 1 S E$. SMRA, saccade to memory-related arrow; SMUA, saccade to memory-unrelated arrow. be-remembered items. The interaction was marginally significant $\left[F(3,33)=3.228, p=.090, \eta_{\mathrm{p}}^{2}=.227\right]$. In post hoc analyses, we performed pairwise comparisons between precision in the SMRA condition and that in the SMUA condition at each set size. The results showed that precision was greater in the SMRA condition than in the SMUA condition at smaller set sizes [set size $1, t(11)=$ $2.150, p=.055$; set size $2, t(11)=2.812, p=.017]$. The difference was not significant at larger set sizes [set size 4 , $t(11)=0.060, p=.954$; set size $6, t(11)=1.205, p=$ $.254]$, possibly because the decreased amplitude of precision for each memory item was lessened, since there were more memory items at larger set sizes.

These results revealed that the total VWM allocated to the memory items was reduced when the saccade target was an extra, not-to-be-remembered item, as compared with when the saccade target was one of the memory items, suggesting that subjects obligatorily allocate VWM to the saccade target, regardless of whether it is irrelevant to or even leads to impairment in the memory task. This finding indicates that the allocation of VWM elicited by saccades is automatic to some extent, rather than perfectly controlled by top-down task setting.

Moreover, for multiitem displays - that is, the number of memory items was more than one $(N>1)$-in the SMRA condition, we compared memory precision for the saccade target and that for the nontargets, which were separately calculated by splitting the data into trials on which the probed item was the saccade target and trials on which it was one of the other items in the sample display. A 2 (type of probe: saccade target vs. non-saccadetarget) $\times 3$ (number of memory items: two, four, or six) within-subjects repeated measures ANOVA showed that memory precision for the saccade target was significantly greater than that for nontargets $[F(1,11)=6.479, p=.027$, $\left.\eta_{\mathrm{p}}^{2}=.371\right]$, which confirmed that there was biased VWM allocation to the saccade target (Bays \& Husain, 2008). In addition, the memory items in the SMUA condition were actually all nontargets for the saccade, and memory precision for them in the multiitem $(N>1)$ displays was similar to that for the nontargets in the SMRA condition $\left[F(1,11)=1.469, p=.251, \eta_{\mathrm{p}}^{2}=.118\right]$, although significantly lower than the precision for the saccade targets in the SMRA condition $\left[F(1,11)=6.781, p=.025, \eta_{\mathrm{p}}^{2}=\right.$ $.381]$. Figure 4 illustrates memory precision for the three types/conditions. The results of this additional analysis demonstrated that precision for the non-saccade-targets was still low even when the task required devoting all the VWM to them (the SMUA condition), thereby further supporting the main suggestion that a part of VWM is allocated to the saccade target obligatorily.

\section{EXPERIMENT 2}

The results of Experiment 1 suggest that VWM will be obligatorily allocated to the not-to-be-remembered saccade target. In other words, it is impossible to perfectly control allocation of VWM when a saccade is made. However, in Experiment 1, the saccade target in the SMUA condition was also an arrow with a random orientation, 


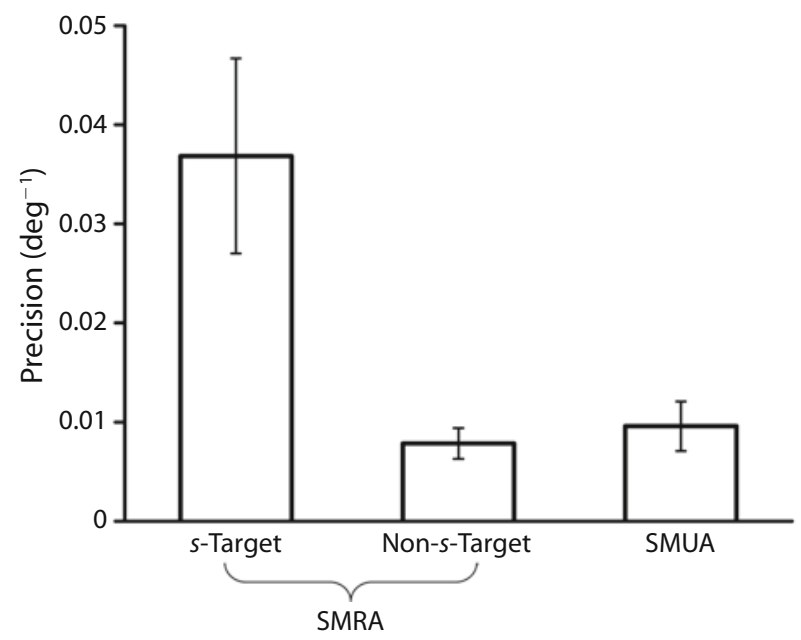

Figure 4. Memory precision in the saccade-to-memory-relatedarrow (SMRA) and saccade-to-memory-unrelated-arrow (SMUA) conditions in Experiment 1 for multiitem displays. The data in the SMRA condition were separated into trials on which the probed item was the saccade ( $s$ ) target and those on which it was one of the non-saccade-targets. Error bars indicate $\pm 1 S E$.

which matched the items in the memory task. According to the biased competition model, the object that matches the description of the information needed by the current task is favored in the visual cortex (Desimone \& Duncan, 1995). It is possible that the saccade target in the SMUA condition in Experiment 1 obtained VWM allocation solely because it matched the items in the memory task. To test this possibility, we changed the saccade target to a cross, which had no connection with the memory task for orientation. By this means, we investigated whether the saccade target still would automatically obtain VWM allocation when it was highly distinguished from the memory items.

\section{Method}

The subjects were the 12 individuals who had participated in Experiment 1 . The stimuli and procedures were the same as those in the SMUA condition in Experiment 1, except that the saccade target was changed to a black cross.

\section{Results and Discussion}

The data from Experiment 2 were compared with those from the SMRA condition in Experiment 1. As in Experiment 1, a 2 (type of saccade target: memory-related arrow vs. memory-unrelated cross) $\times 4$ (number of memory items: one, two, four, or six) within-subjects repeated measures ANOVA yielded significant main effects of both type of saccade target $\left[F(1,11)=6.206, p=.030, \eta_{\mathrm{p}}^{2}=\right.$ $.361]$ and number of memory items $[F(3,33)=14.087$, $\left.p=.002, \eta_{\mathrm{p}}^{2}=.562\right]$, whereas the interaction was not significant $\left[F(3,33)=2.362, p=.150, \eta_{\mathrm{p}}^{2}=.177\right]$.

If task matching alone resulted in the waste of VWM on the saccade target, memory precision in Experiment 2 would be similar to that in the SMRA condition in Experiment 1 . However, actual memory precision still exhibited a significant drop (Figure 3), revealing that VWM will be automatically allocated to a not-to-be-remembered sac- cade target, even if it does not match the information required by the current memory task.

Moreover, we compared memory precision in Experiment 2 and that in the SMUA condition in Experiment 1 in order to investigate the influence of the content of the saccade target on VWM allocation. A 2 (content of saccade target: arrow vs. cross) $\times 4$ (number of memory items) ANOVA revealed that the effect of content was not significant $\left[F(1,11)=1.291, p=.280, \eta_{\mathrm{p}}^{2}=.105\right]$. The effect of number of the memory items was still significant $\left[F(3,33)=16.070, p=.001, \eta_{\mathrm{p}}^{2}=.594\right]$, whereas the interaction was not $\left[F(3,33)=1.039, p=.388, \eta_{\mathrm{p}}^{2}=.086\right]$. The results further prove that the content of the saccade target is not an essential factor in automatic allocation. VWM is allocated to the saccade target in the same manner, regardless of its featural information

\section{EXPERIMENT 3}

In Experiments 1 and 2, we found that VWM was obligatorily allocated to the saccade target. Subjects automatically allocate VWM to the saccade target even when it should be excluded. However, it is not clear whether obligatory allocation is due to a specific interaction between the saccade and VWM or to subjects' general inability to control VWM. Indeed, Vogel et al. (2005) reported that control of VWM is not perfect even when saccades are not made. Low-memory-capacity individuals are predisposed to inefficiently encode and maintain information about irrelevant items. It is possible that most of the subjects who took part in Experiments 1 and 2 were individuals who did not have a general ability to control VWM, resulting in memory allocation to the irrelevant items. To examine this possibility, we tested the same subjects' general controllability of VWM when they did not make a saccade, thereby investigating the cause of the obligatory VWM allocation. If the subjects' memory performance were also impaired by the irrelevant items when remaining fixated, it would mean that the subjects lacked general controllability of VWM. However, if the same subjects were able to effectively exclude the irrelevant items when remaining fixated, it could be concluded that the obligatory VWM allocation to the irrelevant items in Experiments 1 and 2 was elicited by saccades to them.

\section{Method}

The subjects were the 12 individuals who had participated in the preceding experiments. The stimuli and procedures were identical to those in Experiments 1 and 2, except that in this experiment, the subjects were asked to keep fixating the initial cross on each trial and no auditory go signal was sounded. The fixation $(\mathrm{F})$, fixation-with-memory-unrelated-arrow (FMUA), and fixationwith-memory-unrelated-cross (FMUC) conditions were tested in different blocks, and the order of the conditions was counterbalanced across subjects. ${ }^{4}$

\section{Results and Discussion}

We performed a $3 \times 4$ repeated measures ANOVA on memory precision with fixating condition (F, FMUA, or FMUC) and number of memory items (one, two, four, or six) as within-subjects factors. 


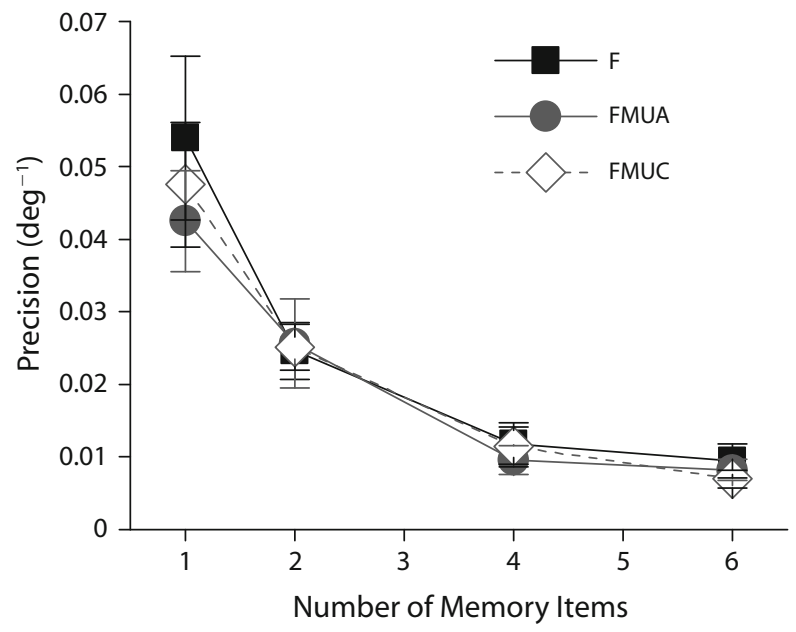

Figure 5. Memory precision as a function of number of memory items and fixating condition in Experiment 3. Error bars indicate $\pm 1 S E$. F, fixation condition; FMUA, fixation-with-memoryunrelated-arrow condition; FMUC, fixation-with-memoryunrelated-cross condition.

Figure 5 shows memory precision of VWM in the three conditions. There was no significant effect of fixating condition $\left[F(2,22)=0.722, p=.449, \eta_{\mathrm{p}}^{2}=.062\right]$, whereas the main effect of number of the memory items remained significant $\left[F(3,33)=28.688, p<.001, \eta_{\mathrm{p}}^{2}=.723\right]$. The interaction was not significant $[F(6,66)=0.371, p=$ $\left..696, \eta_{\mathrm{p}}^{2}=.033\right]$.

These results suggest that the obligatory allocation to the saccade target found in Experiments 1 and 2 could not be explained by the subjects' inability to control VWM, because the subjects could exclude irrelevant items as if the items did not appear in the display when they were fixating throughout. Controllability, however, was impaired when a saccade was made, resulting in automatic VWM allocation to the saccade target, regardless of its irrelevance to the task.

In addition, we compared precision in the $\mathrm{F}$ condition in Experiment 3 with that in the SMRA condition in Experiment 1, using a 2 (condition) $\times 4$ (number of memory items) repeated measures ANOVA. The results yielded a significant main effect of number of memory items $\left[F(3,33)=13.104, p=.003, \eta_{\mathrm{p}}^{2}=.544\right]$ but no effect of condition $\left[F(1,11)=0.007, p=.934, \eta_{\mathrm{p}}^{2}=.001\right]$ and no interaction $\left[F(3,33)=0.994, p=.368, \eta_{\mathrm{p}}^{2}=.083\right]$. The results revealed that the fall in memory precision as a function of number of memory items was similar when there was an intervening saccade and when there was not. The similar precision suggests that a saccade does not reduce the total amount of VWM available, which is consistent with Bays and Husain's (2008) result. Hence, it further supports the hypothesis that the drops in precision in the SMUA condition in Experiments 1 and 2 were due to VWM allocation to the saccade target, and not to any additional VWM cost introduced by saccades per se.

The results above demonstrate that subjects' control of VWM is poor when they make a saccade (Experiment 1), whereas it is great when they remain fixated
(Experiment 3). We further analyzed the data from Experiments 1 and 3 to examine whether they reflected the same kind of control, which varied in strength with different conditions, or whether they reflected different aspects of control of VWM. First, we calculated the decrease in memory precision caused by the memory-unrelated arrow in the two experiments separately: subtracting precision in the FMUA condition from that in the F condition in Experiment 3, and subtracting precision in the SMUA condition from that in the SMRA condition in Experiment 1 . The decrease in memory precision caused by the irrelevant item measured the subjects' ability to control VWM, since a smaller decrease reflected stronger controllability.

Second, we examined individual differences in controllability when the subjects continued to fixate. A one-way ANOVA was performed on the decrease in the amplitude of memory precision caused by the memory-unrelated arrow in Experiment 3, with subject as the factor. The effect of subject was significant $[F(11,36)=2.454, p=$ $\left..021, \eta_{\mathrm{p}}^{2}=.429\right]$, indicating that the ability to control VWM when remaining fixated varied among subjects, which is consistent with Vogel et al.'s (2005) finding.

Then a correlation analysis was conducted between subjects' ability to control VWM when remaining fixated and when making a saccade. The result showed that the correlation between the two conditions above was not significant $(r=.137, p=.670)$, indicating that control of VWM when subjects remained fixated and when they made a saccade was not closely correlated and probably did not reflect the same controllability.

\section{GENERAL DISCUSSION}

In the present research, we examined whether a saccade would elicit obligatory VWM allocation to the saccade target. The results of Experiment 1 showed that, when the saccade target was an extra item that was not required to be remembered (SMUA condition), concurrent memory task performance dropped greatly, as compared with cases in which the saccade target was one of the memory items (SMRA condition). This finding indicates that VWM is obligatorily allocated to the saccade target, even though it is not required. It may be argued that the memoryunrelated saccade target in Experiment 1 was exceptional because it matched the items in the memory task. To test whether task matching was critical for automatic allocation, in Experiment 2, we changed the saccade target to a cross, which was completely different from the to-beremembered arrow(s). The results showed that memory task performance was still significantly poorer than that in the SMRA condition, which further supports the finding in Experiment 1 that VWM allocation to the saccade target is automatic and largely independent of the content of the saccade target. In contrast, the subjects were able to efficiently ignore the memory-unrelated item when fixating throughout (Experiment 3). Thus, allocation of VWM to the memory-unrelated items is indeed elicited by saccades, rather than reflecting a general inability to control VWM allocation. 
Previous research has demonstrated that VWM is largely under volitional control. For example, Yotsumoto and Sekuler (2006) showed that a compound grating stimulus occupying a fixed serial position in a series of study items could be intentionally excluded from memory. Vogel et al. (2005) also reported that when fixating at the center while remembering the items in one hemifield, individuals could selectively exclude irrelevant objects or even irrelevant features of an object from being stored in VWM (Woodman \& Vogel, 2008). Nasr et al. (2008) found that irrelevant objects could be effectively filtered out from VWM after it became clear which objects needed to be retained.

The results of the present research are in accordance with these studies, in that the subjects could selectively allocate VWM to task-related items when fixating throughout (Experiment 3). However, this volitional controllability is greatly impaired when a saccade is made: A portion of VWM will be obligatorily allocated to the saccade target, regardless of its irrelevance to the task.

The obligatory VWM allocation is probably derived from the close link between saccades and VWM-in particular, the important function of VWM in transsaccadic information integration. The saccade target theory of visual stability (Currie, McConkie, Carlson-Radvansky, \& Irwin, 2000; Irwin, McConkie, Carlson-Radvansky, \& Currie, 1994; McConkie \& Currie, 1996) and the visual memory theory (Henderson \& Hollingworth, 2003) suggest that, prior to a saccade, representations are generated and stored in VWM for the saccade target. When the eyes land, the stored representations will be compared with information encoded in the current fixation so as to remap and keep visual stability. As a consequence, changes in the saccade target are easier to detect, as compared with changes in other items (Bays \& Husain, 2008; Henderson \& Hollingworth, 1999b, 2003; Hollingworth \& Henderson, 2002) or even the displacement of the entire background (Currie et al., 2000). The present research further indicates that the saccade target is set as a default memory target. VWM will obligatorily maintain the information of the saccade target. This transsaccadic storage process may be largely automatic and modularized, barely under volitional control, and hardly influenced by the top-down setting of a memory task. Moreover, the storage of the saccade target leads to decreased memory for other items, indicating that this automatic transsaccadic storage shares the same capacity as general short-term storage of visual objects.

The inevitable VWM allocation concomitant with saccades implies that VWM is not a system that is always completely under volitional control or that can even be equated with conscious cognition as typically considered (Atkinson \& Shiffrin, 1971). Probably, VWM is fully under control during fixation, as has been shown in some previous research when subjects continued to fixate. However, under natural, real-life circumstances involving saccades, VWM may be divided into two parts: the volitionally controllable part and the uncontrollable part. The latter possibly equates with or comprises transsaccadic memory, which operates unconsciously to maintain visual stability.
By virtue of this part, a saccade leads to automatic VWM allocation to the saccade target. This is consistent with and complementary to recent research on the functional role of VWM. Hollingworth, Richard, and Luck (2008) demonstrated that an important function of the VWM system is to establish object correspondence across saccades and enable the efficient correction of gaze. They showed that when the features of the saccade target are maintained in VWM, memory-guided gaze correction to the saccade target occurs automatically and largely unconsciously if the eyes fail to land on it. From a different perspective, our present study further demonstrated that, as long as an item is set as the current saccade target, it will be obligatorily maintained in VWM, even against intentional exclusion. Taken together, these results suggest that a part of VWM is so closely coupled with saccades that the saccade target is unlikely to be completely irrelevant for $V W M$ even when it is irrelevant for the memory task.

The two-part division of VWM is further supported by the lack of a correlation between the subjects' ability to control VWM allocation when making a saccade (Experiment 1) and when remaining fixated (Experiment 3). It is probable that the results of Experiment 3, as well as the findings of Vogel et al. (2005), reflected the volitionally controllable part of VWM, whereas the results of Experiment 1 reflected the uncontrollable part of VWM, which is likely to be closely coupled with transsaccadic integration.

Admittedly, automatic storage of a saccade target may lead to seemingly inefficient or uneconomical allocation of VWM under some circumstances. However, saccades are normally used to shift a fixation point toward important or task-dependent stimuli in the natural scene (Henderson \& Hollingworth, 1999a; Rothkopf, Ballard, \& Hayhoe, 2007) so that the stimuli can be precisely and efficiently processed via high-acuity resolution at the fovea. Therefore, in most cases, automatically allocating VWM to the saccade target facilitates cognitive processing effectively and effortlessly.

Up to the present, the nature of the limited information represented in VWM is still a controversial topic. Different models have been proposed to represent VWM, such as the distributed-resource model (Bays \& Husain, 2008, 2009) and the item-limit model (Cowan \& Rouder, 2009; Rouder et al., 2008; Zhang \& Luck, 2008; see also Gao et al., 2009; Xu \& Chun, 2009). However, since the present study focused on the control and operation processes of VWM, it provides no basis for differentiations between the storage models. It is plausible that either a flexible portion of the resources or a discrete slot of VWM is automatically allocated to the saccade target. Further research is needed to reveal the nature of this allocation.

\section{CONCLUSION}

In conclusion, in three experiments, we showed that, concurrent with a saccade, VWM is obligatorily allocated to a saccade target even at the expense of memory task performance. Neither the content of the saccade target nor subjects' general ability to exert control over VWM 
is crucial for the obligatory allocation. Taken together, these findings indicate that VWM consists of an automated component that cooperates with saccades, instead of being completely under top-down control.

\section{AUTHOR NOTE}

The first two authors contributed equally to this study. This research was supported by the Key Project of Humanities and Social Sciences, Ministry of Education (07JZD0029), the National Natural Science Foundation of China (30870765), the Fund of the Ministry of Education for Doctoral Programs in Universities of China (20060335034), and the National Foundation for Fostering Talents of Basic Science (J0630760). We are grateful to three anonymous reviewers for valuable comments and suggestions. We also thank Tao Gao, Junying Liang, Xin Guan, and Jinjin Sun for helpful discussions. Correspondence concerning this article should be addressed to M. Shen, Department of Psychology and Behavioral Sciences, Zhejiang University, Xixi Campus, Hangzhou, 310007, P. R. China (e-mail: mwshen@zju.edu.cn).

\section{REFERENCES}

Atkinson, R. C., \& Shiffrin, R. M. (1971). Control of short-term memory. Scientific American, 225, 82-90.

Ballard, D. H., Hayhoe, M. M., \& Pelz, J. B. (1995). Memory representations in natural tasks. Journal of Cognitive Neuroscience, 7 , 66-80. doi:10.1162/jocn.1995.7.1.66

Bays, P. M., \& Husain, M. (2008). Dynamic shifts of limited working memory resources in human vision. Science, 321, 851-854. doi:10.1126/science. 1158023

Bays, P. M., \& Husain, M. (2009). Response to comment on "Dynamic shifts of limited working memory resources in human vision." Science, 323, 877d. doi:10.1126/science.1166794

BLISS, C. I. (1934). The method of probits. Science, 79, 38-39. doi:10 $.1126 /$ science.79.2037.38

CowAn, N. (2001). The magical number 4 in short-term memory: A reconsideration of mental storage capacity. Behavioral \& Brain Sciences, 24, 87-114. doi:10.1017/S0140525X01003922

Cowan, N., \& Morey, C. C. (2006). Visual working memory depends on attentional filtering. Trends in Cognitive Sciences, 10, 139-141. doi:10.1016/j.tics.2006.02.001

Cowan, N., \& Rouder, J. N. (2009). Comment on "Dynamic shifts of limited working memory resources in human vision." Science, $\mathbf{3 2 3}$, 877c. doi: $10.1126 /$ science. 1166478

Currie, C. B., McConkie, G. W., Carlson-Radvansky, L. A., \& IRwIN, D. E. (2000). The role of the saccade target object in the perception of a visually stable world. Perception \& Psychophysics, 62 , 673-683.

Desimone, R., \& Duncan, J. (1995). Neural mechanisms of selective visual attention. Annual Review of Neuroscience, 18, 193-222. doi:10.1146/annurev.ne.18.030195.001205

Finney, D. J. (1971). Probit analysis (3rd ed.). Cambridge: Cambridge University Press.

Gao, Z., Li, J., Liang, J., Chen, H., Yin, J., \& Shen, M. (2009). Storing fine detailed information in visual working memory-Evidence from event-related potentials. Journal of Vision, 9(7, Art. 17), 1-12. doi: $10.1167 / 9.7 .17$

Griffin, I. C., \& Nobre, A. C. (2003). Orienting attention to locations in internal representations. Journal of Cognitive Neuroscience, 15, 1176-1194. doi:10.1162/089892903322598139

Henderson, J. M., \& Hollingworth, A. (1999a). High-level scene perception. Annual Review of Psychology, 50, 243-271. doi:10.1146/ annurev.psych.50.1.243

Henderson, J. M., \& Hollingworth, A. (1999b). The role of fixation position in detecting scene changes across saccades. Psychological Science, 10, 438-443. doi:10.1111/1467-9280.00183

Henderson, J. M., \& Hollingworth, A. (2003). Eye movements and visual memory: Detecting changes to saccade targets in scenes. Perception \& Psychophysics, 65, 58-71.

Hollingworth, A., \& Henderson, J. M. (2002). Accurate visual memory for previously attended objects in natural scenes. Journal of Experimental Psychology: Human Perception \& Performance, 28, 113-136. doi:10.1037/0096-1523.28.1.113
Hollingworth, A., Richard, A. M., \& LuCK, S. J. (2008). Understanding the function of visual short-term memory: Transsaccadic memory, object correspondence, and gaze correction. Journal of Experimental Psychology: General, 137, 163-181. doi:10.1037/0096 $-3445.137 .1 .163$

Honda, H. (1991). The time courses of visual mislocalization and of extraretinal eye position signals at the time of vertical saccades. Vision Research, 31, 1915-1921. doi:10.1016/0042-6989(91)90186-9

HoNDA, H. (1999). Modification of saccade-contingent visual mislocalization by the presence of a visual frame of reference. Vision Research, 39, 51-57. doi:10.1016/S0042-6989(98)00134-5

Irwin, D. E., McConkie, G. W., Carlson-Radvansky, L. A., \& CuRRIE, C. (1994). A localist evaluation solution for visual stability across saccades. Behavioral \& Brain Sciences, 17, 265-266.

Lappe, M., Awater, H., \& Krekelberg, B. (2000). Postsaccadic visual

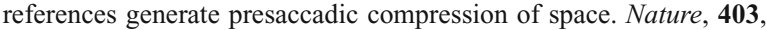
892-895. doi:10.1038/35002588

Lavie, N. (2005). Distracted and confused?: Selective attention under load. Trends in Cognitive Sciences, 9, 75-82. doi:10.1016/j.tics.2004 .12 .004

Lavie, N., Hirst, A., De Fockert, J. W., \& Viding, E. (2004). Load theory of selective attention and cognitive control. Journal of Experimental Psychology: General, 133, 339-354. doi:10.1037/0096-3445 .133 .3 .339

Luck, S. J., \& Vogel, E. K. (1997). The capacity of visual working memory for features and conjunctions. Nature, 390, 279-281. doi: $10.1038 / 36846$

McConkie, G. W., \& Currie, C. B. (1996). Visual stability across saccades while viewing complex pictures. Journal of Experimental Psychology: Human Perception \& Performance, 22, 563-581. doi:10.1037/0096-1523.22.3.563

McNicol, D. (1972). A primer of signal detection theory. London: Allen \& Unwin.

Melcher, D., \& Colby, C. L. (2008). Trans-saccadic perception. Trends in Cognitive Sciences, 12, 466-473. doi:10.1016/j.tics.2008.09.003

Nasr, S., Moeeny, A., \& Esteky, H. (2008). Neural correlate of filtering of irrelevant information from visual working memory. PLoS ONE, 3, e3282. doi:10.1371/journal.pone.0003282

Olesen, P. J., Macoveanu, J., Tegner, J., \& Klingberg, T. (2007). Brain activity related to working memory and distraction in children and adults. Cerebral Cortex, 17, 1047-1054. doi:10.1093/cercor/ bh1014

Prime, S. L., Tsotsos, L., Keith, G. P., \& Crawford, J. D. (2007). Visual memory capacity in transsaccadic integration. Experimental Brain Research, 180, 609-628. doi:10.1007/s00221-007-0885-4

Ross, J., Morrone, M. C., \& Burr, D. C. (1997). Compression of visual space before saccades. Nature, 386, 598-601. doi:10.1038/386598a0

Ross, J., Morrone, M. C., GoldberG, M. E., \& Burr, D. C. (2001). Changes in visual perception at the time of saccades. Trends in Neurosciences, 24, 113-121. doi:10.1016/S0166-2236(00)01685-4

Rothkopf, C. A., Ballard, D. H., \& HaYhoe, M. M. (2007). Task and context determine where you look. Journal of Vision, 7(14, Art. 16), 1-20. doi:10.1167/7.14.16

Rouder, J. N., Morey, R. D., Cowan, N., Zwilling, C. E., Morey, C. C., \& Pratte, M. S. (2008). An assessment of fixed-capacity models of visual working memory. Proceedings of the National Academy of Sciences, 105, 5975-5979. doi:10.1073/pnas.0711295105

Sreenivasan, K. K., \& JHA, A. P. (2007). Selective attention supports working memory maintenance by modulating perceptual processing of distractors. Journal of Cognitive Neuroscience, 19, 32-41. doi:10.1162/jocn.2007.19.1.32

Vogel, E. K., McCollough, A. W., \& Machizawa, M. G. (2005). Neural measures reveal individual differences in controlling access to working memory. Nature, 438, 500-503. doi:10.1038/nature04171

Wickens, T. D. (2002). Elementary signal detection theory. New York: Oxford University Press.

Woodman, G. F., \& Vogel, E. K. (2008). Selective storage and maintenance of an object's features in visual working memory. Psychonomic Bulletin \& Review, 15, 223-229. doi:10.3758/PBR.15.1.223

Xu, Y., \& Chun, M. M. (2009). Selecting and perceiving multiple visual objects. Trends in Cognitive Sciences, 13, 167-174. doi:10.1016/ j.tics.2009.01.008

Yotsumoto, Y., \& Sekuler, R. (2006). Out of mind, but not out of 
sight: Intentional control of visual memory. Memory \& Cognition, 34, 776-786.

ZHANG, W. W., \& LUCK, S. J. (2008). Discrete fixed-resolution representations in visual working memory. Nature, 453, 233-235. doi:10.1038/ nature 06860

\section{NOTES}

1. Here, it is necessary to distinguish between irrelevant for the memory task and irrelevant for VWM. In this article, when we refer to a saccade target as irrelevant or memory unrelated, we mean that it is irrelevant for the memory task. In fact, the key question in the present study is whether a saccade target is completely irrelevant for VWM when it is irrelevant for the memory task.

2. Bays and Husain (2008) assessed memory allocation for two kinds of features, orientation and location, and demonstrated similar results.
However, the location task may be slightly complicated. Visual space perception is distorted around the onset of a saccade (Ross et al., 2001). In particular, presaccadic displacement (including magnitude and direction) strongly rests on an object's onset time relative to the saccade, its position within a visual field, and the presence of visual references (Honda, 1991, 1999; Lappe, Awater, \& Krekelberg, 2000; Ross, Morrone, \& Burr, 1997). Therefore, we rather preferred the clear and less confused orientation task for the present research.

3. A probit model is a popular specification of a generalized linear model in statistics, which is widely available in software for statistics and probability modeling. The probit (Bliss, 1934; Finney, 1971) function is the quantile function associated with the standard normal distribution (Gaussian distribution), which provides a basis for important types of regression.

4. Each subject completed all three experiments in a counterbalanced order, so as to avoid a possible practice effect.

\section{APPENDIXA}

\section{Additional Analyses of the Methodology (1)}

In the present study, we fixed the interval between the auditory go signal and the offset of the sample display to match the estimate of average saccade latency for each subject and then screened the data according to the actual saccade latency on each trial. It is necessary to account for the effectiveness of this method.

First, distributions of actual saccade latencies were compared across the three saccade conditions/experiments. We depict the distribution of actual saccade latencies in Experiments 1 and 2 in Figure A1. The consistency of the distribution across conditions/experiments was examined with a chi-square test. The results showed that, in the separate conditions/experiments, the actual distributions of saccade latencies did not differ significantly $\left[\chi^{2}(41)=50.82, p=.140\left(\right.\right.$ SMUA vs. SMRA); $\chi^{2}(41)=19.26, p=.999($ SMUA vs. Experiment 2$) ; \chi^{2}(41)=$ $41.54, p=.447$ (Experiment 2 vs. SMRA)].

Second, the percentage of trials that met the four criteria (the included rate) was consistent across the three conditions/experiments, as revealed by a one-way repeated measures ANOVA on the included rate, with conditions/experiments as the within-subjects factor $\left[F(2,22)=1.220, p=.314, \eta_{\mathrm{p}}^{2}=.100\right]$. The overall included rate was acceptable (56\%, on average) in view of the stringent fourfold criterion for trial inclusion.

Third, the subjects' difference on the included rate was examined across the three conditions/experiments. A one-way repeated measures ANOVA on the included rate, with subjects as the factor, showed that there were significant differences among subjects $\left[F(11,24)=2.266, p=.046, \eta_{\mathrm{p}}^{2}=.509\right]$. Starting from Lavie's load theory (Lavie, 2005; Lavie, Hirst, de Fockert, \& Viding, 2004), it was possible that the included rate influenced the suppression strength of the task-irrelevant stimulus among subjects, because the subjects who were more likely to fail the criteria might devote more attention to the task requirements, which led to a higher cognitive control load and weaker suppression of the irrelevant stimulus. To test whether the main results were confounded by the included rate, we examined memory performance for the subjects with different included rates. Twelve subjects were divided into high-included-rate and low-included-rate groups by using a median split of their included rate. The two groups differed significantly in average included rate [64\% vs. $48 \% ; t(10)=3.560, p=.005]$. We calculated the strength of suppressing the task-irrelevant stimulus through subtracting memory precision in the SMUA condition from that in the SMRA condition in Experiment 1. A 2 (group: high-included-rate group or low-included-rate group) $\times 4$ (number of memory items: one, two, four, or six) repeated measure ANOVA was performed on the suppression strength of task-irrelevant stimuli, with group as the between-subjects factor and number of memory items as the within-subjects factor. The results showed that neither group nor number of memory items yielded a significant effect $\left[F(1,10)=0.488, p=.519, \eta_{\mathrm{p}}^{2}=.043 ; F(3,30)=3.129, p=.098\right.$, $\left.\eta_{\mathrm{p}}^{2}=.238\right]$. There was no interaction either $\left[F(3,30)=0.663, p=.457, \eta_{\mathrm{p}}^{2}=.062\right]$.

The results indicated that the subjects who differed in the included rate did not differ in the suppression strength of the task-irrelevant stimuli. We surmised that perhaps the subjects with low included rates did not actually devote much more attention to the task requirements in the present study, since there was no feedback after each trial.

Fourth, we examined the consistency of the experimental effect across trials with saccades of different latencies. One might be concerned that the saccades that started after the appearance of the blank had turned into memory-guided saccades, which were qualitatively different from the deferred saccades expected from the experimental settings. And perhaps it was this memory-guided task that led to the VWM allocation toward the saccade targets. To examine this possibility, a further analysis was performed. We split the data into two sections: the trials with short-latency saccades (Section 1) and the trials with long-latency saccades (Section 2). The shortlatency saccades were defined as saccades that were launched no later than $100 \mathrm{msec}$ after the blanking of the sample display. Because the saccade latencies in the present experiments were typically longer than $200 \mathrm{msec}$, these short-latency saccades could not be memory-guided saccades in response to the blank but were deferred saccades in response to the go signal. We performed a 2 (section: 1 vs. 2 ) $\times 2$ (condition: SMRA vs. SMUA) repeated measures ANOVA on memory precision. If VWM allocation to the saccade targets was elicited by the memory-guided task in the trials with long-latency saccades, the experimental effect would show up only 

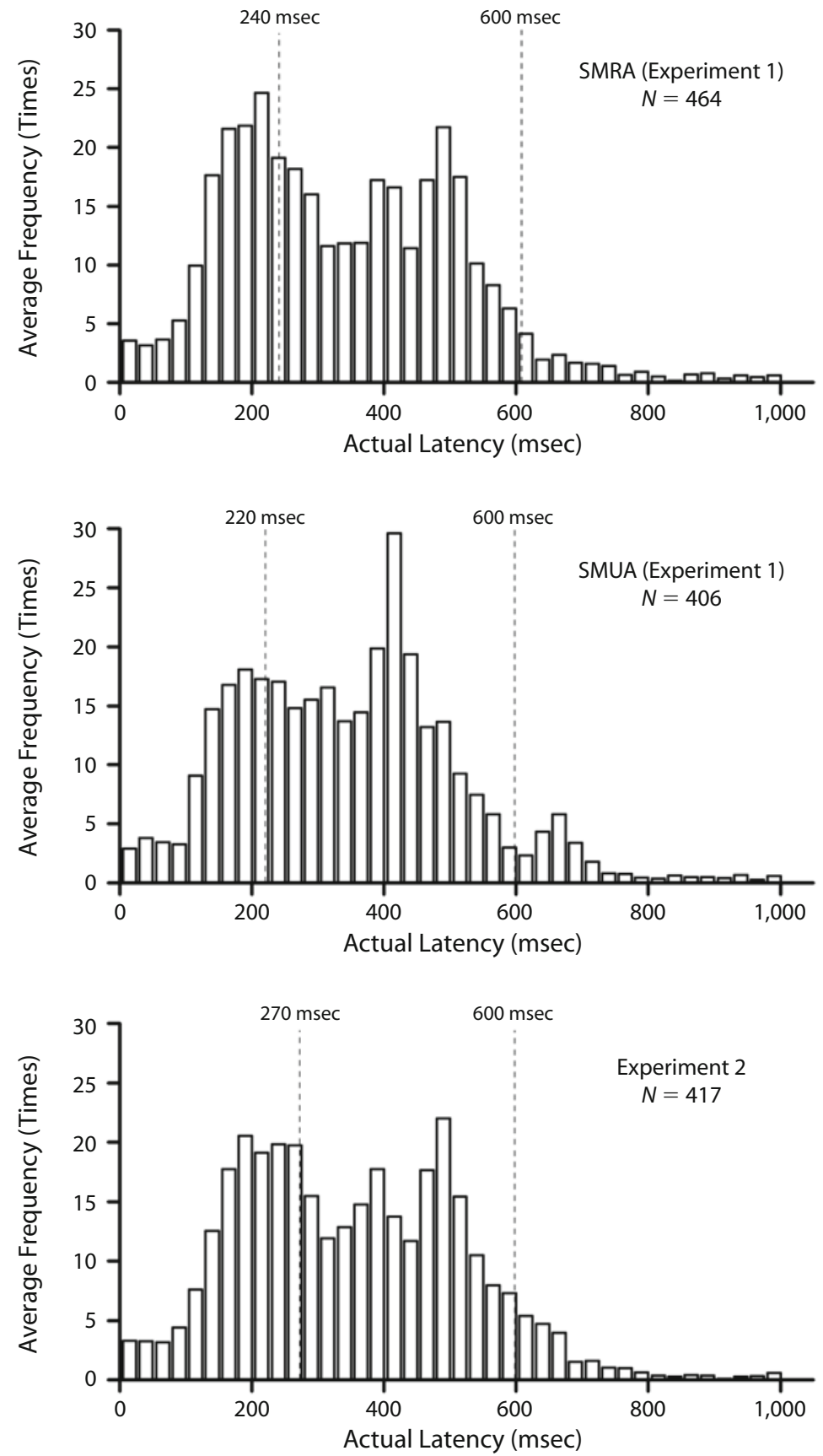

Figure A1. Distributions of actual saccade latencies in the saccade-to-memoryrelated-arrow (SMRA) and saccade-to-memory-unrelated-arrow (SMUA) conditions in Experiment 1 and in Experiment 2. $N$ represents the average number of total trials in each condition/experiment. The origin point on the horizontal axis corresponds to the onset time of the auditory go signal, at which time the subjects are required to initiate a saccade. The two dashed lines depict Trial Inclusion Criteria 2 and 3. Trials on which the saccade latency was less than zero (i.e., the subjects launched a saccade before the go signal) or longer than 1,000 msec are not shown in the figure. 


\section{APPENDIXA (Continued)}

in Section 2. That is, there would be an interaction between section and condition, and precision in the SMRA condition would be greater than that in the SMUA condition only in Section 2, but not in Section 1. However, the results showed a significant main effect of condition $\left[F(1,11)=5.811, p=.035, \eta_{\mathrm{p}}^{2}=.346\right]$, with no interaction $\left[F(1,11)=0.056, p=.817, \eta_{\mathrm{p}}^{2}=.005\right]$ and no main effect of section $\left[F(1,11)=2.936, p=.115, \eta_{\mathrm{p}}^{2}=.211\right]$. The analysis demonstrated that precision was consistently greater in the SMRA condition than that in the SMUA condition, regardless of whether the trials had short-latency saccades or long-latency saccades (Figure A2). Therefore, the allocation to the saccade targets was not elicited on the trials with long-latency saccades due to a qualitative change in the saccade task but was elicited on all the trials because of obligatory VWM allocation toward saccade targets. The two sections of trials were homogeneous for the analysis of VWM allocation. It is appropriate to include all these trials and merge them together in data analyses, as we did in our study.

Taken together, the examinations above indicate that the method is effective. Basically, it achieved the same effect as online saccade detection, with no impact on the main results in the present study.

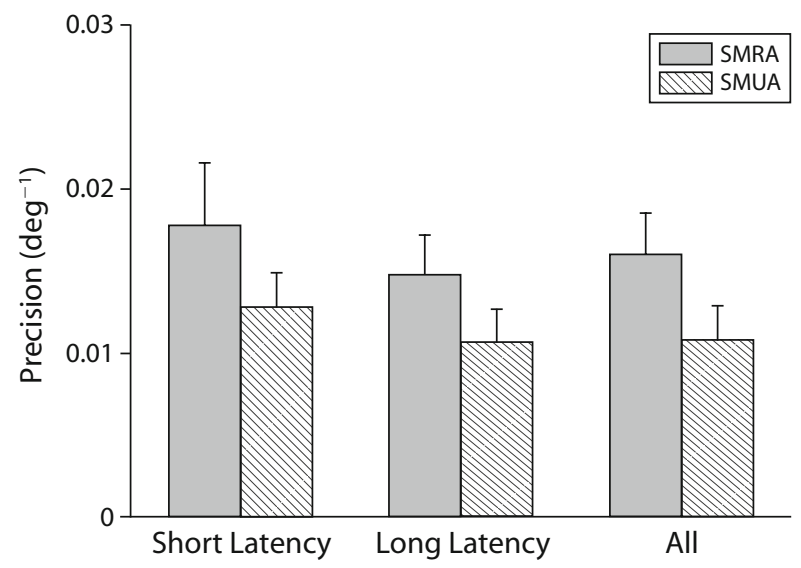

Figure A2. Memory precision in the saccade-to-memory-relatedarrow (SMRA) and saccade-to-memory-unrelated-arrow (SMUA) conditions on the trials with short-latency saccades (left column), on the trials with long-latency saccades (middle column), and across all the trials (right column). Error bars indicate $\pm 1 S E$.

\section{Additional Analyses of the Methodology (2)}

The focus of the present study was the allocation of VWM under saccade conditions, rather than the saccade per se. Therefore, saccade accuracy was not treated as a dependent variable. There was no feedback for the accuracy of the saccades during the process of the experiments either. We hold that saccade accuracy did not confound the experimental effect of the present study. The reasons are as follows.

First, the question of the present study was whether saccades elicit obligatory VWM allocation to the saccade target. If the effect of the saccade target did not show up, it is possible that the saccades were so inaccurate that the saccade target set by the task actually was not differentiated from other items. However, the present results revealed that VWM was allocated to the saccade, which indicated that the saccades were accurate enough for the present study.

Second, there is the question of whether the effort for accurate saccades confounded the results in the present study. Theoretically, there might be a trade-off between the memory task and saccade task, so that the lower memory precision in the SMUA condition in Experiment 1 and in Experiment 2 was possibly due to a larger cost for the saccade. However, in the present study, the relative positions of the saccade target in the SMUA condition and in Experiment 2 were fixed (at the center of the memory array display area), whereas they varied from trial to trial in the SMRA condition. Therefore, the cost for the saccade task should be smaller in the SMUA condition and Experiment 2, because of less uncertainty about the landing point. If it is determined by the cost for saccades, memory performance in these conditions/experiments should have been better than (or similar to) that in the SMRA condition. However, the results turned out to be the opposite. Hence, the possibility can be ruled out that memory precision decreased in the SMUA condition in Experiment 1 and in Experiment 2 because of different costs for saccades.

When these analyses are taken together, the saccades in this paradigm are ensured to be accurate enough for the present study. Besides, the method is suitable for investigating the present topic without introducing any confusion. 


\section{APPENDIX B \\ Additional Analyses of the Results of Probit Regression}

In the major analyses, the influence of the different conditions for VWM allocation was examined by changes in memory precision. The precision was calculated by the reciprocal of the standard deviation of the Gaussian distribution. To present the results thoroughly, the goodnesses of fit of the psychometric functions and the means of the Gaussian distribution also needed to be mentioned.

Overall, the goodnesses of fit of the psychometric functions were acceptably good. There were 288 fitted probit functions in total ( 6 conditions/experiments $\times 12$ subjects $\times 4$ set sizes), and the $p$ values of the chi-square tests were larger than .05 in 266 of them.

We performed a one-way repeated measures ANOVA on the means of the probit regression functions, with condition/experiment as the within-subjects factor (Experiment 1, SMRA, SMUA; Experiment 2; Experiment 3, F, FMUA, FMUC). The results showed that the main effect of condition/experiment was not significant $\left[F(5,55)=1.251, p=.289, \eta_{\mathrm{p}}^{2}=.102\right]$, indicating that there were no systematic effects for the means of the psychometric functions across all conditions/experiments. The results further support the major findings of the present study by showing that there was no change of discrimination bias across the conditions/experiments, whereas subjects' memory precision varied systematically.

(Manuscript received March 31, 2009;

revision accepted for publication December 13, 2009.) 